\title{
Spatio-temporal variations of the flood mitigation service of ecosystem under different climate scenarios in the Upper Reaches of Hanjiang River Basin, China
}

\author{
WANG Pengtao ${ }^{1,2}$, 'ZHANG Liwei ${ }^{1}$, LI Yingjie ${ }^{3}$, JIAO Lei ${ }^{1}$, WANG Hao ${ }^{1}$, \\ YAN Junping ${ }^{1}$, LÜ Yihe ${ }^{4}$, FU Bojie ${ }^{4}$ \\ 1. Department of Geography, School of Geography and Tourism of Shaanxi Normal University, Xi'an 710119, China; \\ 2. School of Tourism \& Research Institute of Human Geography, Xi'an International Studies University, Xi'an \\ 710128, China; \\ 3. Center for Systems Integration and Sustainability, Michigan State University, East Lansing, MI 48823, USA; \\ 4. State Key Laboratory of Urban and Regional Ecology, Research Center for Eco-Environmental Sciences, \\ CAS, Beijing 100085, China
}

\begin{abstract}
Extreme rainstorm and the subsequent flood increasingly threaten the security of human society and ecological environment with aggravation of global climate change and anthropogenic activity in recent years. Therefore, the research on flood mitigation service (FMS) of ecosystem should be paid more attention to mitigate the risk. In this paper, we assessed FMS in the Upper Reaches of Hanjiang River (URHR), China from 2000 to 2014 using the Soil Conservation Service Curve Number (SCS-CN) model, and further simulated the future FMS under two climate scenarios (in 2020 and 2030). The results reveal that the FMS presented a fluctuating rising trend in the URHR from 2000 to 2014 . The FMS in southern URHR was higher than that of northern URHR, and the change rate of FMS in the upstream of URHR (western URHR) was higher than the downstream of URHR (eastern URHR). The future FMS under scenarios of Medium-High Emissions (A2) and Medium-Low Emissions (B2) will decrease consistently. As land use/land cover changes in the URHR are negligible, we concluded that the change in FMS was mainly driven by climate change, such as storm and runoff. Our study highlights that climate scenarios analysis should be incorporated into the assessment of hydrologic-related services to facilitate regional water resources management.
\end{abstract}

Keywords: ecosystem services; scenario analysis; climate change; SCS-CN model; Hanjiang River

Received: 2017-05-15 Accepted: 2017-12-08

Foundation: Natural Science Basic Research Plan in Shaanxi Province of China, No.2017JQ4009; National Natural Science Foundation of China, No.41601182, No.41471097; National Social Science Foundation of China, No.14AZD094; Key Project of Chinese Ministry of Education, No.15JJD790022; The National Key Research and Development Plan of China, No.2016YFC0501601; The Science and Technology Service Network Initiative Project of Chinese Academy of Sciences, No.KFJ-STS-ZDTP-036; Fundamental Research Funds for the Central University, No.GK201703053

Author: Wang Pengtao (1988-), PhD, specialized in climate change and ecosystem services. E-mail: wnpengtao@126.com "Corresponding author: Zhang Liwei (1985-), PhD and Associate Professor, specialized in landscape ecology and ecosystem services. E-mail: zlw@snnu.edu.cn 


\section{Introduction}

Hydrological services are critical for sustaining ecosystem structure, ecosystem process and region ecological environment security (Castello and Macedo, 2016; Sun et al., 2016). These services can be divided into four categories: diverted and in situ water supply, flood mitigation service, water-related cultural services, and water-associated supporting services (Brauman et al., 2007), among which the flood mitigation service (FMS) has drawn much attention because of the increasingly intensification of global climate change and extreme storm event in recent years (Barbedo et al., 2014; Watson et al., 2016; Sonter et al., 2017). FMS is one of the key flood regulation services (Bagstad et al., 2011, 2014; Sturck et al., 2014), which denotes the capability of ecosystem to reduce and retain floodwater to avoid flood damages to downstream populations by vegetation, soil and other components of ecosystem (Carvalho-Santos et al., 2016; Watson et al., 2016; Sonter et al., 2017). Compared with human-dominated water conservancy project, the natural ecosystem plays a more positive and effective role in flood mitigation (Sturck et al., 2014; Barth and Döll, 2016), and has less negative impacts on biodiversity protection and ecological environment (Zhang et al., 2010; Sturck et al., 2014; Keesstra et al., 2018).

In fact, flood mitigation is a comprehensive hydrological process, which is composed of flood interception by canopy, litter, flood storage in soil, and storm runoff, etc. (Zhang et al., 2010; Barth and Döll, 2016; Kim et al., 2016). Thus, vegetation and soil, even artificial land in terrestrial ecosystem, all have the potential to mitigate the flood (Nedkov and Burkhard, 2012). However, most of the previous studies focused on the FMS of wetland and soil (Marsooli et al., 2016; Ouyang et al., 2016; Pappalardo et al., 2016; Watson et al., 2016; Liu et al., 2017), but few assessed the whole natural ecosystem. For instance, Ouyang et al. (2016) adopted the empirical method between the available water storage capacity and area to assess the flood mitigation capacity of wetland. Jiang et al. (2007) estimated the water subtraction quantity of wetland soils within the Momoge Reserve. As far as we know, Nelson et al. (2009) adopted the InVEST model to assess FMS in the terrestrial ecosystem at a fine spatial resolution, however, data availability of single storm event limits the application of this model to large-scale areas. Zhang et al. (2010) and Kim et al. (2016) grouped these hydrological process as interception, stem flow, litter interception and water storage, whereas, it is very complicated to calculate these ES and is difficult to establish precise mathematical model. Promisingly, Fu et al. (2013) developed a method to assess FMS based on watershed runoff model, i.e., the SCS-CN model (Li et al., 2015; Lin et al., 2017). This SCS-CN model takes account of multiple watershed characteristics, such as soil, land use, hydrologic condition and antecedent moisture condition (AMC), and it has great potential to evaluate FMS at a broad scale (Mishra et al., 2012; Chen et al., 2017; Lal et al., 2017). In addition, current research seldom analyzed the temporal dynamics of FMS, let alone the variations under different climate changing scenarios (Fu et al., 2015). The lacking of systematical understanding of FMS variations may weaken the decision-making for regional flood management.

In this study, the Upper Reaches of Hanjiang River basin (URHR) is taken as study area. URHR is the water source of the middle route of South-to-North Water Transfer Project (SNWTP) in China. The study of hydrologic ecosystem services and water resources in URHR, not only concerns the local ecological water use safety and the socio-economic de- 
velopment, but also provides benefits for the sustainability of lower reaches of Hanjiang River and national water recipient areas of SNWTP (Chen et al., 2007; Li et al., 2016). Besides, rapid changes in land use and climate have increasingly influenced the ecosystem services (ES), therefore, it is necessary to look into the plausible future change of ES. In recent environment research, the scenarios analysis is widely adopted as a vital and effective approach providing possible descriptions of future change in climate or land use based on reasonable assumption (Feng et al., 2016; Runting et al., 2017; Wang et al., 2017). The integration of these plausible and reasonable descriptions to ES research will help stakeholders better understand the processes and response mechanisms of ecosystem to environmental change and anthropogenic activity (Scholes 2016; Thom et al., 2017; Yuan et al., 2017), as well as provide scientific reference for targeted preventive measures in face of the future flood risk in URHR.

Based on the FMS model and climate change scenarios analysis, this paper aims to: (1) analyze the historical spatio-temporal variations of FMS from 2000 to 2014 and the future variations in the years of 2020 and 2030 under two climate scenarios in URHR; (2) discuss the major driving factors of FMS variations; and (3) discuss the implication of climate scenarios analysis on FMS management. This research may contribute to a better understanding of the relationship between climate change and ecosystem services (ES), and help facilitate watershed water resources management.

\section{Materials and methodology}

\subsection{Study area}

Hanjiang River is the largest branch of Yangtze River in China, and it is also the water source of the middle route of South-to-North Water Diversion Project in China (Chen et al., 2007; Li et al., 2016). The Upper Reaches of Hanjiang River basin is located between $105.85^{\circ}-111.60^{\circ} \mathrm{E}$ and $31.02^{\circ}-34.48^{\circ} \mathrm{N}$, with a basin area of $62384 \mathrm{~km}^{2}$, and extends 652 $\mathrm{km}$ from the west to the east in Shaanxi province (Figure 1). This basin is characterized by sub-tropical monsoon climate, and precipitation concentrates in summer and autumn, with frequent storms and continuous rain (Chen et al., 2007). The average annual temperature ranges from $12^{\circ} \mathrm{C}$ to $18^{\circ} \mathrm{C}$, and the average annual precipitation ranges from 653 to 1183 mm (Li et al., 2017; Wang et al., 2017). Topographically, the northern border of URHR is formed by the Qinling Mountains, and southern border is formed by the Daba Mountain and Micang Mountain. Hanjiang River flows through these mountains. The URHR is known as a key natural ecological zone in central China, and flood disasters frequently occur in this region, due to the natural condition (i.e., steep slopes and large river bed gradient) and subtropical humid monsoon climate. This poses great threats to the human well-being in the middle and lower reaches of Hanjiang.

\subsection{Datasets and methodology}

\subsubsection{Flood mitigation service model}

Soil Conservation Service curve number (SCS-CN) model, is one of the most popular methods for evaluating the surface runoff volume in a single rain event (Mishra et al., 2012). 
It accounts for watershed characteristics of runoff yield and has been adopted by engineers and practitioners in various regions under different climatic conditions (Yang et al., 2015; Hooshyar and Wang, 2016; Liu and Li, 2017). Integrating SCSCN model into FMS assessment can be helpful for the spatially-explicit evaluation of storm runoff and flood mitigation magnitude in large watershed.

In the study, we apply SCS-CN model to estimate FMS in two steps:

(1) Evaluating storm runoff: the theoretical base of SCS-CN is water balance and the two basic assumptions in

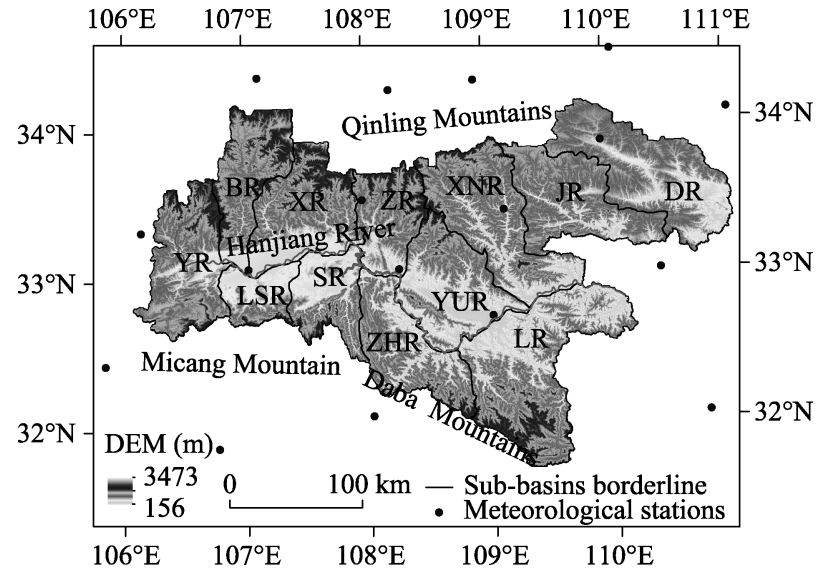

Figure 1 Location and 12 sub-basins of the Upper Reaches of Hanjiang River (URHR), China. These sub-basins are Yanghe River (YR), Baohe River (BR), Xuhe River (XR), Ziwuhe River (ZR), Xunhe River (XNR), Jinqian River (JR), Danjiang River (DR), Lengshuihe River (LSR), Shuangmahe River (SR), Zhuhe River (ZHR), Yuehe River (YR), Lanhe River (LR), respectively.

$$
\begin{gathered}
P=I_{a}+F+Q \\
\frac{Q}{P-I_{a}}=\frac{F}{S} \\
I_{a}=\lambda S
\end{gathered}
$$

where $P$ represents the rainfall $(\mathrm{mm}), I_{a}$ represents the initial abstraction $(\mathrm{mm}), F$ represents cumulative infiltration ( $\mathrm{mm}), Q$ represents the direct surface runoff $(\mathrm{mm}), S$ represents the potential maximum retention $(\mathrm{mm})$ and $\lambda$ represents the initial abstraction coefficient (generally, $\lambda$ is set as 0.2 in this work).

Surface runoff can be calculated as follows:

$$
\begin{gathered}
Q=\left\{\begin{array}{cc}
\frac{\left(P-I_{a}\right)^{2}}{\left(P-I_{a}+S\right)}, & P \geqslant I_{a} \\
0, & P<I_{a}
\end{array}\right. \\
S=\frac{25400}{C N}-254
\end{gathered}
$$

Particularly, substituting $P_{s}$ (representing storm rain) into formulas SCS-CN model, $Q_{s}$ (representing storm runoff) can be calculated.

$$
\left\{\begin{array}{cc}
Q_{s}=\frac{\left(P_{s}-I_{a}\right)^{2}}{\left(P_{s}-I_{a}+S\right)}, & P_{s} \geqslant I_{a} \\
Q_{s}=0, & P_{s}<I_{a}
\end{array}\right.
$$

In the model, $C N$ is a comprehensive variable that accounts for hydrologic soil group (HSG), land use and antecedent moisture condition (AMC), and the parameters can be looked up from the table in the National Engineering Handbook (USDA, 1985). The table of $C N$ value was built up in ArcGIS 10.2. The land use data of the URHR in 2000, 2005 and 2010 were selected to represent the land use condition in each period: 2000-2004, 2005-2009 and 2010-2014 respectively. 
(2) The flood mitigation service can be evaluated by using water balance equation (Fu et al., 2013):

$$
F_{M}=P_{s}-Q_{s}
$$

where $F_{M}$ represents flood mitigation (mm). Substituting $P_{s}$ and $Q_{s}$ in Eq. (6) into Eq. (7), the $F_{M}$ can be put as follows:

$$
F_{M}=\left(S_{e v}-\frac{\left(S_{e v}-0.25\right)^{2}}{S_{e v}+0.85}\right) \times \text { storm_days }
$$

where $S_{e v}$ represents single rainstorm event ( $\left.\mathrm{mm}\right)$, storm_days represents the storm days per year.

\subsubsection{Climate change scenarios setting}

Scenarios are stories that describe possible futures about socio-economic, technological and environmental conditions, etc. (Moss et al., 2010). Applied in climate change research, this approach can promote understanding of complex interactions of climate conditions, human activities and ecosystems (Moss et al., 2010; Liang et al., 2017), and has great value in better providing references for targeted options for the regional decision-making and ecosystem management (Fu et al., 2015). General Circulation Models (GCMs) are the most popular methods in climate change research, studying the future scenarios and dynamic mechanism of climate change through mathematical equations. However, the GCM outputs are too coarse for the regional climate study, so we downscaled the model to better fit for our study (Yang et al., 2017). In this study, the automated regression-based statistical downscaling tool (ASD) was chosen to set the climate change scenarios in URHR (Hessami et al., 2008). The model is widely used because of its preferable simulation effect and simple operation (Guo et al., 2012; Lu et al., 2016). In the simulation process of ASD, three forms of data were used:

(1) Daily precipitation data (from 2000 to 2014) of meteorological stations in the URHR. This dataset was derived from National Meteorological Information Center of China Meteorological Administration (http://data.cma.cn/). The storm events were selected from the meteorological data.

(2) NCEP/NCAR reanalysis data at the spatial scale of $1^{\circ} \times 1^{\circ}$ from 1991 to 2001 . The data were derived from National Centers for Environmental Prediction and National Center for Atmospheric Research.

(3) Climate change scenarios (from 1961 to 2099) of the Hadley Centre Coupled Model Version 3 (HadCM3) output data under Special Report on Emissions Scenarios (SRES) A2 and B2 scenarios at the spatial scale of $3.75^{\circ} \times 2.5^{\circ}$ (Johns et al., 2003). This dataset was derived from Hadley Centre for Climate Prediction and Research. The A2 scenario represents a Medium-High Emissions world with more rapid population growth but less rapid economic growth, and B2 scenario represents a Medium-Low Emissions world with slower population growth, economic and social sustainable development (Nakicenovic et al., 2000; Walz et al., 2014). ASD method was applied to obtain the future storm runoff and FMS in 2020 and 2030, representing relatively near future, in which the FMS can be comparable with the current situation and targeted preventive measures can be carried out timely.

Before setting future climate scenarios of precipitation and FMS, the model performance had been evaluated with the calibration process in the period of 1961-1975 and validation process in the period of 1976-1990. Specifically, in evaluation process, the Nash-Sutcliffe 
efficiency coefficient (NS) and the coefficient of determination $\left(R^{2}\right)$ had been used to evaluate the residual between measured precipitation data and the simulation results, with the value $0.76(\mathrm{NS})$ and $0.78\left(R^{2}\right)$ in calibration period, $0.83(\mathrm{NS})$ and $0.84\left(R^{2}\right)$ in validation period, which indicated the model is considered acceptable and accurate in local area (Lu et al., 2016). Thus, with the output from ASD model, the future storm, storm runoff and FMS can be obtained.

\section{Results}

\subsection{Temporal and spatial variations of flood mitigation service}

During the period of 2000-2014, the annual mean FMS in the URHR was $101.93 \mathrm{~mm}$. The annual FMS ranged from $34.42 \mathrm{~mm}$ in 2001 to $203.98 \mathrm{~mm}$ in 2011, with a fluctuating rising trend of $1.97 \mathrm{~mm} / \mathrm{yr}$. This change trend was consistent with the results of China's first national ecosystem assessment (2000-2010) (Ouyang et al., 2016). Besides, during the 15-year period, there were evident fluctuating inter-annual variations of FMS, and the FMS reversed abruptly in 2011. Thus, the change of FMS could be divided into two phases: in the first phase, FMS continuously increased at a rate of $6.20 \mathrm{~mm} / \mathrm{yr}$ from 2000 to 2010, while subsequently, FMS dramatically decreased at a rate of $-48.99 \mathrm{~mm} / \mathrm{yr}$ from 2011 to 2014 .

Spatial pattern of FMS revealed an apparent gradation from southwest to northeast (Figure 2a). In the southern URHR, the values of FMS were high, especially in the LSR, SR and ZHR region, where the FMS values were above $130 \mathrm{~mm}$. By contrast, the values of FMS in the north were far less, and the values in the DR, BR, XNR and JR were less than $80 \mathrm{~mm}$ (Figure 2c). In most regions of the URHR, FMS had increased since 2000, while in the northwestern, DR was the only area where FMS decreased. Meanwhile, the change rates of FMS in the west and the east of the URHR were significantly different (Figure 2b). Specifically, the change rates of FMS in the eastern URHR (downstream of URHR) were relatively
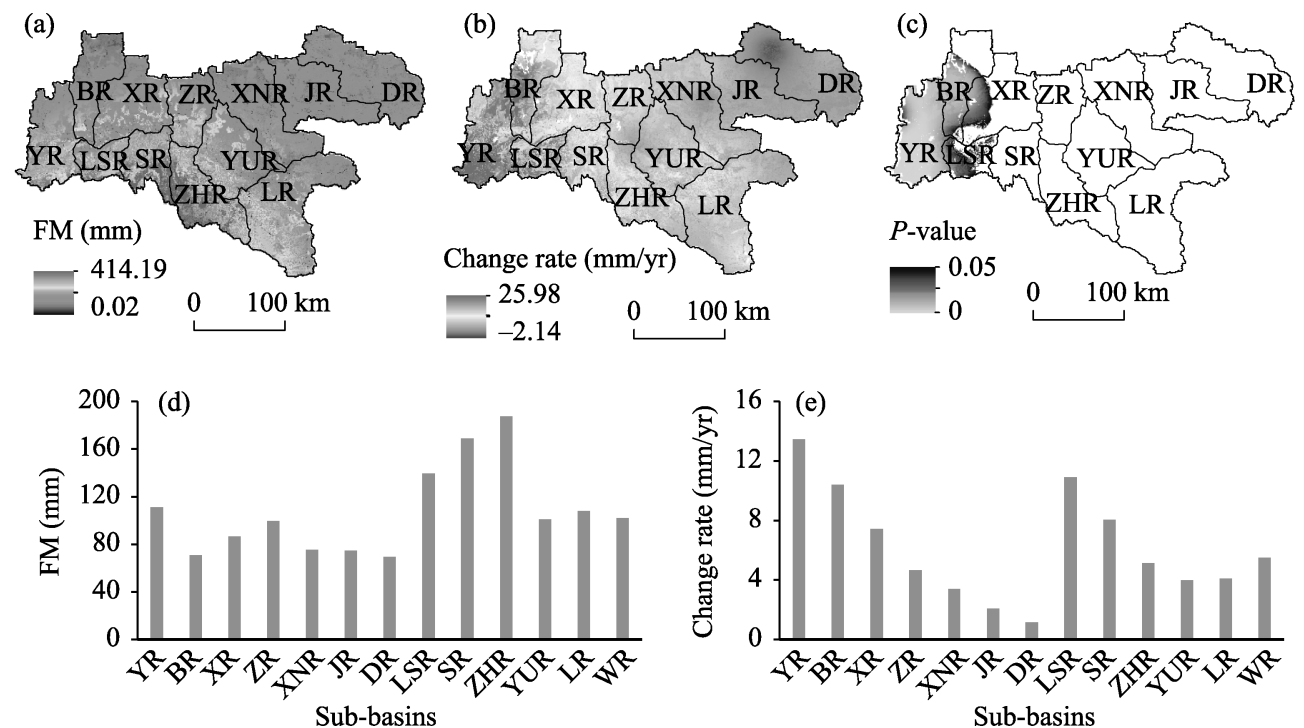

Figure 2 Spatial patterns of annual FMS (a), change rate (b), the significant level (c) and sub-basins distribution of annual average (d) and change rate (e) of flood mitigation service in the URHR. In (d) and (e), WR represents the whole region (URHR). 
low, for example, the change rates in DR, JR and XNR were under $4 \mathrm{~mm} / \mathrm{yr}$, while in the western URHR (upstream of URHR) the change rates were relatively high, the values in YR, BR and LSR were all above $10 \mathrm{~mm} / \mathrm{yr}(P<0.05)$.

\subsection{Flood mitigation service in different climate scenarios}

In the climate scenarios of $\mathrm{A} 2$ and $\mathrm{B} 2$ in 2020 and 2030, the mean FMS $\left(\mathrm{FMS}_{\mathrm{A} 2-2020}\right.$, $\mathrm{FMS}_{\mathrm{A} 2-2030}, \mathrm{FMS}_{\mathrm{B} 2-2020}$ and $\mathrm{FMS}_{\mathrm{B} 2-2030}$ ) will be 82.15, 63.43, 64.37 and $60.97 \mathrm{~mm}$, respectively. In comparison with historical annual mean FMS $(101.93 \mathrm{~mm})$, the FMS in the four scenarios will all decrease. Besides, the future FMS $\left(\mathrm{FMS}_{\mathrm{A} 2-2020}, \mathrm{FMS}_{\mathrm{A} 2-2030}, \mathrm{FMS}_{\mathrm{B} 2-2020}\right.$ and $\mathrm{FMS}_{\mathrm{B} 2-2030}$ ) in each sub-basin will all be lower than the historical mean value.

(a) A2-2020

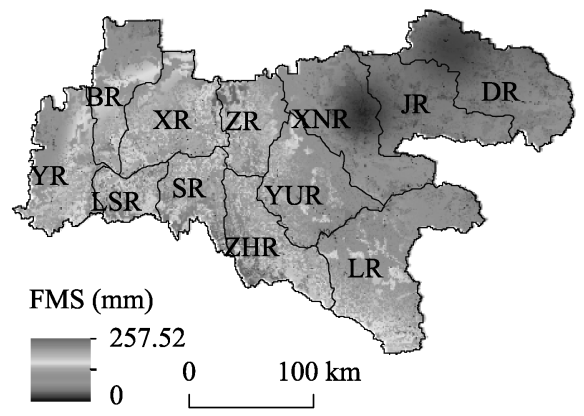

(c) $\mathrm{A} 2-2030$

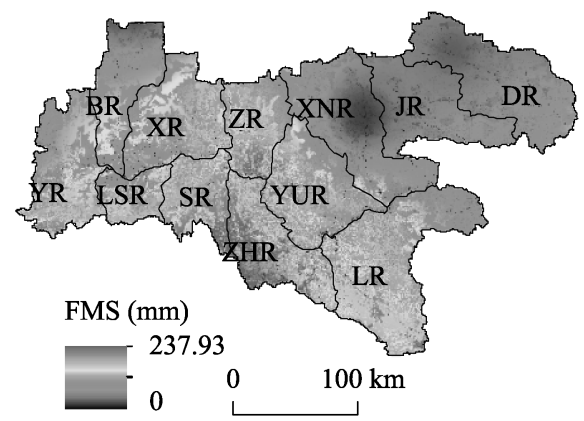

(b) B2-2020

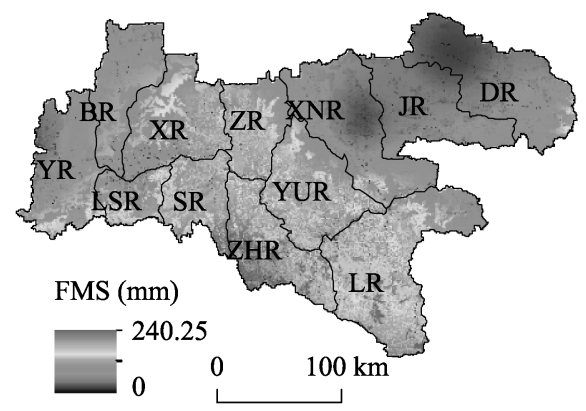

(d) B2-2030

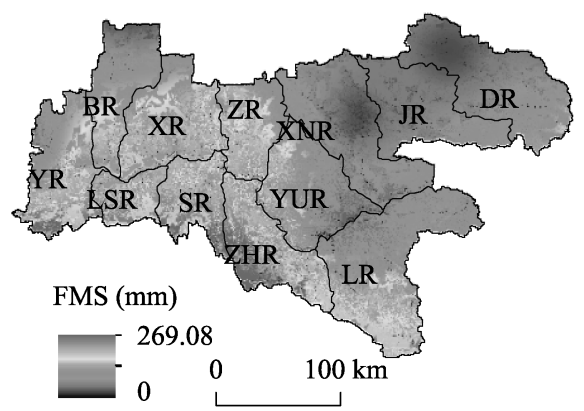

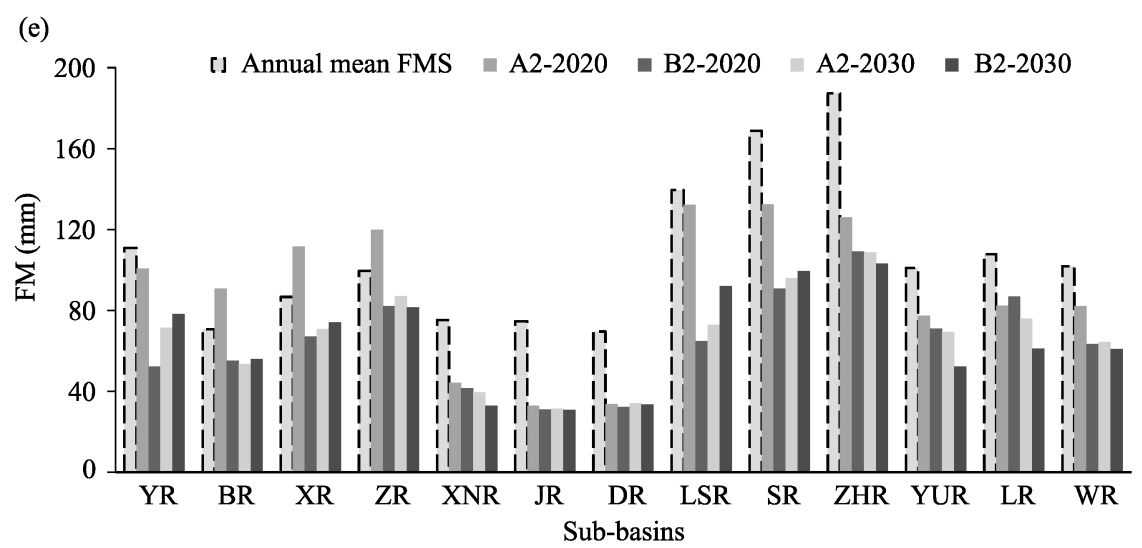

Figure 3 The flood mitigation map under climate change scenarios in the URHR: A2-2020 (a), B2-2020 (b), A2-2030 (c), B2-2030 (d) and sub-basins distribution in scenarios (e). In (e), WR represents the whole region (URHR). 
The FMS in the same SRES scenario varies by year: in SRES A2, the FMS in 2030 will be lower than value in 2020 in each sub-basin; in SRES B2, in upstream of URHR (YR, BR, XR, LSR and SR), FMS in $2030\left(\mathrm{FMS}_{\mathrm{B} 2-2030}\right)$ will be higher than value in $2020\left(\mathrm{FMS}_{\mathrm{B} 2-2020}\right)$, and in downstream of URHR, the FMS in $2030\left(\mathrm{FMS}_{\mathrm{B} 2-2030}\right)$ will be lower than value in 2020 $\left(\mathrm{FMS}_{\mathrm{B} 2-2020}\right)$. In addition, the FMS in the future years varies by SRES scenarios: in 2020, the scenario value for $\mathrm{A} 2\left(\mathrm{FMS}_{\mathrm{A} 2-2020}\right)$ will be nearly higher than value for $\mathrm{B} 2\left(\mathrm{FMS}_{\mathrm{B} 2-2020}\right)$ in each sub-basin; in 2030, the scenario value for $\mathrm{A} 2\left(\mathrm{FMS}_{\mathrm{A2}-2030}\right)$ will be lower than value for B2 $\left(\mathrm{FMS}_{\mathrm{B} 2-2030}\right)$ in upstream of URHR (YR, BR, XR, LSR and SR), and in downstream of URHR, the scenario value for $\mathrm{A} 2\left(\mathrm{FMS}_{\mathrm{A} 2-2030}\right)$ will be higher than value for $\mathrm{B} 2\left(\mathrm{FMS}_{\mathrm{B} 2-2030}\right)$.

In sum, in downstream of URHR, the future FMS will continuously decrease from 2020 to $2030\left(\mathrm{FMS}_{2020}>\mathrm{FMS}_{2030}\right)$ under the same scenarios and the future FMS will also be lower under a Medium-Low Emissions scenario $\left(\mathrm{FMS}_{\mathrm{B} 2}\right)$ than a Medium-High Emissions $\left(\mathrm{FMS}_{\mathrm{A} 2}\right)$ in the same year. However, the variations of scenarios value in upstream of URHR will be unstable between years or between SRES scenarios.

\section{Discussion}

\subsection{Driving factors of the FMS variations}

Land use/land cover (LULC) change and climate change are key factors that impact the runoff, consequently, influence the FMS (Piao et al., 2007; Fu et al., 2015). The LULC change has a major impact on runoff and flood mitigation processes, such as canopy interception, depression detention, infiltration, and so on. However, the LULC in the URHR showed limited change during 2000-2014 because of the mountainous environment and backward economic development. According to the LULC transfer matrix (Table 1), the land use in URHR presented little change. In terms of land use types, the woodland, artificial land (e.g., land for construction) and wetland rose gradually, while the cropland, bare land and grassland declined slowly. In general, LULC change will affect major runoff and flood mitigation processes, such as canopy interception, depression detention, infiltration, etc. However, the change in artificial land, wetland, bare land and grassland was feeble (all less than $50 \mathrm{~km}^{2}$ ), which limited their effect on the local FMS variations. With the implementation in ecological projects, such as "Grain for Green Project" in Qinling-Daba Mountains (Liu et al., 2016), the vegetation coverage in URHR increase gradually, thus the increasing of woodland might

Table 1 The transfer matrix of land use/land cover in URHR from 2000 to $2014\left(\mathrm{~km}^{2}\right)$

\begin{tabular}{lccccccc}
\hline \multicolumn{1}{c}{ Land cover } & Grassland & Wetland & Cropland & Artificial land & Bare land & Woodland & Decrease \\
\hline Grassland & 367.25 & 1.25 & 0 & 0 & 0 & 0 & 1.25 \\
Wetland & 0 & 328 & 1.25 & 0 & 6.25 & 0 & 7.5 \\
Cropland & 0 & 11.06 & 12629.19 & 41.81 & 2.44 & 351.38 & 406.69 \\
Artificial land & 0 & 0 & 0 & 401.69 & 0 & 0 & 0 \\
Bare land & 0 & 24.56 & 0 & 0 & 245.69 & 0 & 24.57 \\
Woodland & 0 & 0 & 0 & 0 & 2.5 & 48412.57 & 2.5 \\
Increase & 0 & 36.88 & 1.25 & 41.81 & 11.19 & 351.38 & - \\
Net change & -1.25 & 29.38 & -405.44 & 41.81 & -13.38 & 348.88 & - \\
Relative change (\%) & -0.34 & 8.56 & -3.02 & 10.41 & -4.54 & 0.72 & - \\
\hline
\end{tabular}


facilitate the increase in FMS (Fu et al., 2013). However, the relative change in woodland only occurred in the limited areas (e.g., some river basins and low hills area in URHR), being less than $1 \%$ of the whole area. As a result, during 2000-2014, the land use in URHR showed little change, and thus has little effect on local FMS variations.

In addition, we compared the temporal change and spatial patterns of the storm, runoff and FMS in the URHR to assess their similarities and differences. During 2000-2014, the storm, runoff and FMS increased at rates of $0.52,1.97$ and $2.49 \mathrm{~mm} / \mathrm{yr}$, with the similar fluctuant change trends and characteristics. The FMS was highly correlated with the storm and runoff: the correlation coefficient between FMS and storm was $0.96(P<0.01)$, and the correlation coefficient between FMS and runoff was $0.97(P<0.01)$. Additionally, the FMS shared a similar distribution pattern with storm and runoff in change trend (see Figure 2(b), Figure 4(b) and Figure 4(c)). The change rates of FMS, storm and runoff in upstream of URHR were all higher than that in downstream of URHR. Besides, similar spatial distribution patterns of these three factors' change rates also could be found in sub-basins distribution. As for FMS change in different SRES scenarios, we found that the FMS all showed apparent decreasing trend, which were similar to the decreasing trend of storm and runoff. The A2 and B2 scenarios are different perspectives of socio-economic situation which are set based on the deduced global greenhouse gas emission, therefore, the different scenarios lead to different variations of FMS in 2020 and 2030. However, the future FMS under scenarios of A2 and B2 in 2020 and 2030 all show decreasing trend, though they differ in the magnitude of variations.

According to both the historical and future scenario analysis of FMS and its potential driving factors' change, it can be concluded that the FMS in URHR was mainly determined by climate change, such as storm and runoff.

\subsection{Implications of scenario analysis for mapping FMS}

Ecosystem supply FMS by reducing and retaining floodwater in forest canopy, leaf litter and soil to avoid flood damages to downstream populations, and the FMS is one of the key hydrological services. However, the increasingly intensification of climate change and human activities can lead to degradation of the FMS, increasingly threatening the local water resources security and ecological stability. In FMS research, flood risk zonation can be executed according to relative risk grade of different areas, Priority Areas for protecting FMS can be designated in local area (Zhang et al., 2017), and some potential problems in regional water crisis could be prevented or mitigated in the future.

The provision of ES depends on biophysical conditions of ecosystem (Sturck et al., 2015). The ecosystem has been and will continue be increasingly influenced by anthropogenic land use change and climate change (Burkhard et al., 2012). Given this, a long-term study on the ecosystem change can help better understand the mechanism and future trend of the ES change (Li et al., 2017). For example, in this study, the FMS increased with fluctuations from 2000 to 2010, while FMS decreased dramatically from 2010 to 2013, which revealed the internal instability of ecosystem under the changing environment. Our study also found that storm rain had a significant perturbation on FMS. Thus, the analysis of historical change can help identify driving factors of FMS, and further provide theoretical basis for scenario analysis. For instance, the climate change is the main factor that influences on FMS, so the 

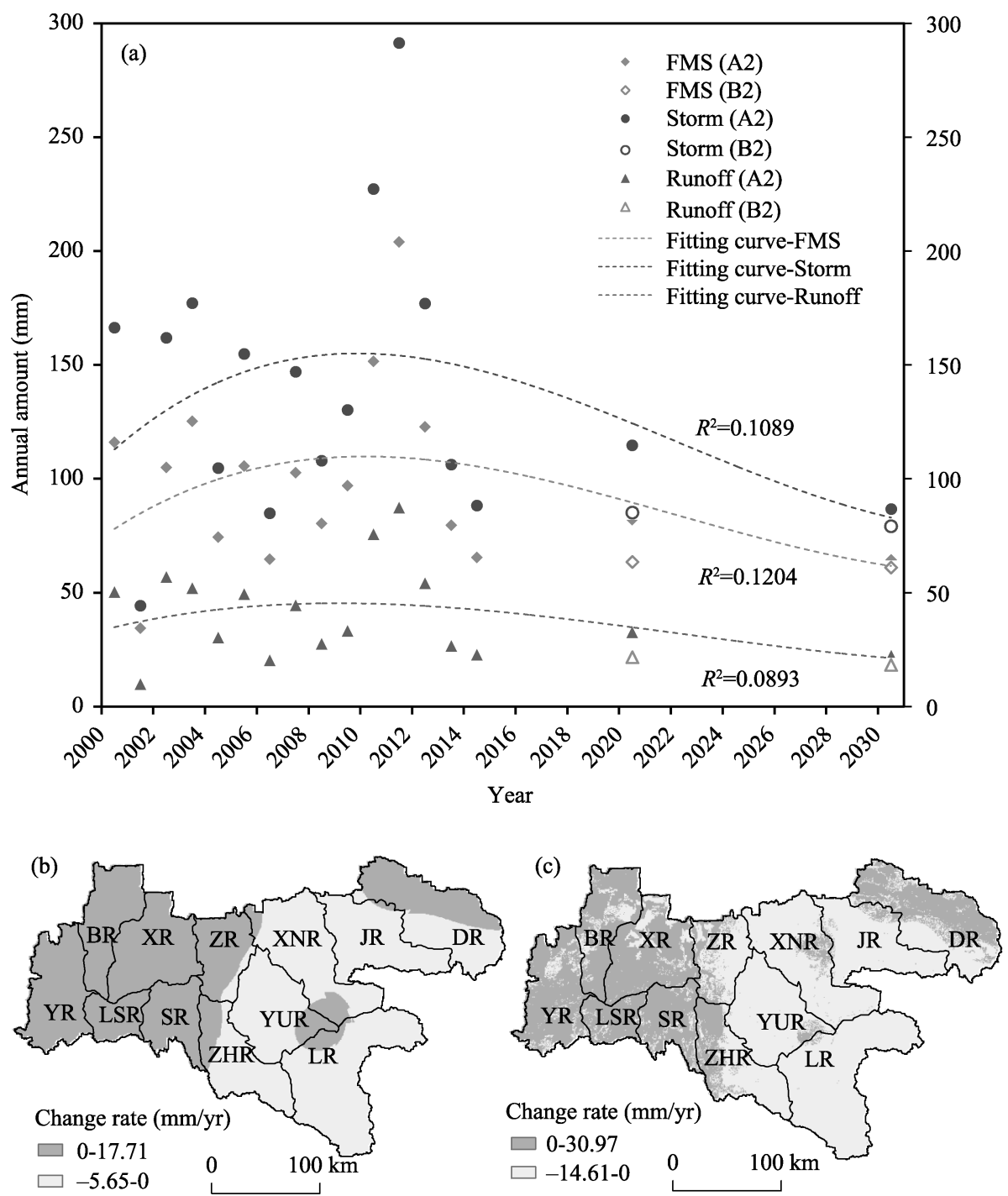

(d)

(e)
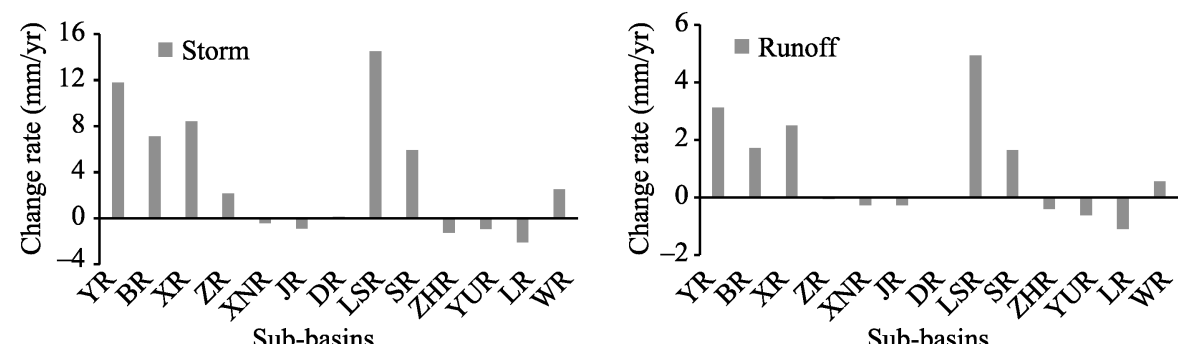

Figure 4 Temporal variations of storm and runoff (a), spatial variations of storm change slope (b), spatial variations of runoff change slope (c), storm change slope of sub-basins (d) and runoff change slope of sub-basins (e). In (d) and (e), WR represents the Whole Region (URHR).

climate scenario was chosen to simulate the future FMS. The driving forces chosen in this study are only a special example, and in general, ES is driven by multi-factor in complicated and non-linear process. To find out the dominant factors, more comprehensive methods are needed. 
Scenario analysis helps stakeholders consider the possible futures change of ES. After knowing the potential impact of future environmental change, stakeholders could develop adapting strategies to prevent regional water resources risks (Leng et al., 2015; Gosling et al., 2016; Popp et al., 2017). For instance, in the URHR, our study showed that the FMS will decrease in 2020 and 2030 under climate scenarios of A2 and B2. With this knowledge, we can suggest that there would be less flood risk in the URHR, and the stakeholder may invest moderate funding to defense risk. Besides, by integrating historical change and scenario analysis into ES assessment, we provide a useful framework to understand the whole dynamic variations of ES. After controlling for some variables and contrast changes of variables, the dominant factor and possible magnitude of the effect of dominant factor on ESs can be identified (Popp et al., 2017).

There are also some uncertainties in the process of FMS evaluation, though we carefully deal with the models. First, future climate changes scenarios provide a possible but not completely accurately description of future climate change (Leng et al., 2015). In this study, the impacts of historical and future climate change on the ecosystem services is the main content of research, thus there is only one climate model (HadCM3) involved in. In further study about the construction of exhaustive ecosystem services assessment, more diverse climate change models and scenarios should be taken into consideration. It could be helpful for the stakeholders to find out more suitable measures and put forward a more targeted policy in protection of natural ecosystem. In addition, the key parameter $\mathrm{CN}$ in the SCS-CN model is sensitive to the local land use change, micro-topography and local climate change, and may affect the runoff calculation to some extent (Fu et al., 2013). On the small-scale regional studies, the local land use micro-change should be taken into account. Furthermore, the land use change scenario should be considered in the future studies, especially in regions where land use changes rapidly. As discussed above, the land use change has a remarkable hydrological effect on ecosystem (Carvalho-Santos et al., 2016; Zuo et al., 2016), and Fu et al. (2015) also emphasized that more attention should be paid to the link between ES and land use. In our study, the land use of URHR showed little change during 2000-2014, so we did not model the land use scenario. However, in a constantly and dramatically land use changing region, the role of land use change in the FMS should not be neglected.

\section{Conclusions}

Assessing and mapping of flood mitigation service (FMS) are critical for regional water resources and flood risk management. Based on the SCS-CN model and climate scenario analysis, we analyzed the historical spatio-temporal variations and future variations of FMS in the URHR, China. The main conclusions are as follows:

FMS showed a fluctuating rising trend during the period of 2000-2014, and FMS reversed abruptly in 2011, thus dividing the period into two phases with apparently different trends: FMS increased from 2000 to 2010, while decreased from 2011 to 2014. Spatially, the FMS in southern URHR was higher than that of northern URHR, and the change rate of FMS in the upstream of URHR was higher than downstream of URHR. The future FMS under scenarios of A2 and B2 in 2020 and 2030 will decrease in comparison with the historical annual mean FMS (2000-2014). For each sub-basin, compared with 2020, the FMS in 2030 will decrease further. Besides, the difference between A2 and B2 scenarios is quite small. 
The slight land use changes in the URHR have feeble impacts on the FMS, while the runoff and storm change have a significant influence on the FMS. We concluded that the climate change played a key role in the flood mitigation in the URHR.

Our study suggests that both historical and scenario analysis are vital for better understanding the ecological process, and practically provides scientific reference for government and stakeholders to make targeted and purposeful measures in watershed water resources management.

\section{Acknowledgements}

We are grateful to Dr. Zhang Jie from the Institute of Geographic Sciences and Natural Resources Research, Chinese Academy of Sciences, who provided essential help in the simulation and validation of climate change scenarios in this paper.

\section{References}

Bagstad K J , Villa F, Batker D et al., 2014. From theoretical to actual ecosystem services: Mapping beneficiaries and spatial flows in ecosystem service assessments. Ecology and Society, 19(2): 64.

Bagstad K J, Villa F, Johnson G W et al., 2011. ARIES: Artificial Intelligence for Ecosystem Services: A guide to models and data, version 1.0 Beta. The ARIES Consortium, Bilbao, Spain. [online] URL: http://www.ariesonline.org/docs/ARIESModelingGuide1.0.pdf.

Barbedo J, Miguez M, van der H D et al., 2014. Enhancing ecosystem services for flood mitigation: A conservation strategy for peri-urban landscapes? Ecology and Society, 19(2): 54.

Barth N C, Döll P, 2016. Assessing the ecosystem service flood protection of a riparian forest by applying a cascade approach. Ecosystem Services, 21, Part A: 39-52.

Brauman K A, Daily G C, Duarte T K et al., 2007. The nature and value of ecosystem services: An overview highlighting hydrologic services. Annual Review of Environment and Resources, 32: 67-98.

Burkhard B, Kroll F, Nedkov S et al., 2012. Mapping ecosystem service supply, demand and budgets. Ecological Indicators, 21: 17-29.

Carvalho-Santos C, Nunes J P, Monteiro A T et al., 2016. Assessing the effects of land cover and future climate conditions on the provision of hydrological services in a medium-sized watershed of Portugal. Hydrological Processes, 30(5): 720-738.

Castello L, Macedo M N, 2016. Large-scale degradation of Amazonian freshwater ecosystems. Global Change Biology, 22(3): 990-1007.

Chen H, Guo S, Xu C Y et al., 2007. Historical temporal trends of hydro-climatic variables and runoff response to climate variability and their relevance in water resource management in the Hanjiang Basin. Journal of Hydrology, 344(3/4): 171-184.

Chen H, Liang Z, Liu Y et al., 2017. Integrated remote sensing imagery and two-dimensional hydraulic modeling approach for impact evaluation of flood on crop yields. Journal of Hydrology, 553(Suppl. C): 262-275.

Feng X M, Fu B J, Piao S et al., 2016. Revegetation in China's Loess Plateau is approaching sustainable water resource limits. Nature Climate Change, 6(11): 1019-1022.

Fu B, Wang Y K, Xu P et al., 2013. Mapping the flood mitigation services of ecosystems: A case study in the Upper Yangtze River Basin. Ecological Engineering, 52: 238-246.

Fu B J, Zhang L W, Xu Z H et al., 2015. Ecosystem services in changing land use. Journal of Soils and Sediments, 15(4): 833-843.

Gosling S N, Arnell N W, 2016. A global assessment of the impact of climate change on water scarcity. Climatic Change, 134(3): 371-385.

Guo J, Chen H, Xu C Y et al., 2012. Prediction of variability of precipitation in the Yangtze River Basin under the climate change conditions based on automated statistical downscaling. Stochastic Environmental Research and 
Risk Assessment, 26(2): 157-176.

Hessami M, Gachon P, Ouarda T B M J et al., 2008. Automated regression-based statistical downscaling tool. Environmental Modelling \& Software, 23(6): 813-834.

Hooshyar M, Wang D, 2016. An analytical solution of Richards' equation providing the physical basis of SCS curve number method and its proportionality relationship. Water Resources Research, 52(8): 6611-6620.

Jiang M, Lu X G, Xu L S et al., 2007. Flood mitigation benefit of wetland soil: A case study in Momoge National Nature Reserve in China. Ecological Economics, 61(2/3): 217-223.

Johns T C, Gregory J M, Ingram W J et al., 2003. Anthropogenic climate change for 1860 to 2100 simulated with the HadCM3 model under updated emissions scenarios. Climate Dynamics, 20(6): 583-612.

Keesstra S, Nunes J, Novara A et al., 2018. The superior effect of nature based solutions in land management for enhancing ecosystem services. Science of The Total Environment, 610 (Suppl. C): 997-1009.

Kim B, Kim H, Kim H, 2016. A framework for pricing the loss of regulating ecosystem services caused by road construction. KSCE Journal of Civil Engineering, 20(7): 2624-2631.

Lal M, Mishra S K, Pandey A et al., 2017. Evaluation of the Soil Conservation Service curve number methodology using data from agricultural plots. Hydrogeology Journal, 25(1): 151-167.

Leng G, Tang Q, Rayburg S, 2015. Climate change impacts on meteorological, agricultural and hydrological droughts in China. Global and Planetary Change, 126(Suppl. C): 23-34.

Li J, Liu C, Wang Z et al., 2015. Two universal runoff yield models: SCS vs. LCM. Journal of Geographical Sciences, 25(3): 311-318.

Li Y, Xiong W, Zhang W et al., 2016. Life cycle assessment of water supply alternatives in water-receiving areas of the South-to-North Water Diversion Project in China. Water Research, 89: 9-19.

Li Y, Zhang L, Qiu J et al., 2017. Spatially explicit quantification of the interactions among ecosystem services. Landscape Ecology, 32(6): 1181-1199.

Li Y, Zhang L, Yan J et al., 2017. Mapping the hotspots and coldspots of ecosystem services in conservation priority setting. Journal of Geographical Sciences, 27(6): 681-696.

Liang S, Hurteau M D, Westerling A L, 2017. Response of Sierra Nevada forests to projected climate-wildfire interactions. Global Change Biology, 23(5): 2016-2030.

Lin W, Yang F, Zhou L et al., 2017. Using modified Soil Conservation Service curve number method to simulate the role of forest in flood control in the upper reach of the Tingjiang River in China. Journal of Mountain Science, 14(1): 1-14.

Liu C, Li Y, 2017. GIS-based dynamic modelling and analysis of flash floods considering land-use planning. International Journal of Geographical Information Science, 31(3): 481-498.

Liu J, Wang X, Zhang B et al., 2017. Storm flood risk zoning in the typical regions of Asia using GIS technology. Natural Hazards, 87(3): 1691-1707.

Liu X, Zhu X, Pan Y et al., 2016. Vegetation dynamics in Qinling-Daba Mountains in relation to climate factors between 2000 and 2014. Journal of Geographical Sciences, 26(1): 45-58.

Lu Y, Qin X S, Xie Y J, 2016. An integrated statistical and data-driven framework for supporting flood risk analysis under climate change. Journal of Hydrology, 533: 28-39.

Marsooli R, Orton P M, Georgas N et al., 2016. Three-dimensional hydrodynamic modeling of coastal flood mitigation by wetlands. Coastal Engineering, 111: 83-94.

Mishra S K, Pandey A, Singh V P, 2012. Special Issue on Soil Conservation Service Curve Number (SCS-CN) Methodology Introduction. Journal of Hydrologic Engineering, 17(11): 1157-1157.

Moss R H, Edmonds J A, Hibbard K A et al., 2010. The next generation of scenarios for climate change research and assessment. Nature, 463(7282): 747-756.

Nakicenovic N, Alcamo J, Davis G et al., 2000. Special Report on Emissions Scenarios: A Special Report of Working Group III of the Intergovernmental Panel on Climate Change. Cambridge, UK: Cambridge University Press.

Nedkov S, Burkhard B, 2012. Flood regulating ecosystem services: Mapping supply and demand, in the Etropole municipality, Bulgaria. Ecological Indicators, 21: 67-79.

Nelson E, Mendoza G, Regetz J et al., 2009. Modeling multiple ecosystem services, biodiversity conservation, com- 
modity production, and tradeoffs at landscape scales. Frontiers in Ecology and the Environment, 7(1): 4-11.

Ouyang Z, Zheng H, Xiao Y et al., 2016. Improvements in ecosystem services from investments in natural capital. Science, 352(6292): 1455-1459.

Pappalardo S E, Otto S, Gasparini V et al., 2016. Mitigation of herbicide runoff as an ecosystem service from a constructed surface flow wetland. Hydrobiologia, 774(1): 193-202.

Piao S, Ciais P, Huang Y et al., 2010. The impacts of climate change on water resources and agriculture in China. Nature, 467(7311): 43-51.

Piao S, Friedlingstein P, Ciais P et al., 2007. Changes in climate and land use have a larger direct impact than rising $\mathrm{CO}_{2}$ on global river runoff trends. Proceedings of the National Academy of Sciences of the United States of America, 104(39): 15242-15247.

Popp A, Calvin K, Fujimori S et al., 2017. Land-use futures in the shared socio-economic pathways. Global Environmental Change, 42(Suppl. C): 331-345.

Runting R K, Bryan B A, Dee L E et al., 2017. Incorporating climate change into ecosystem service assessments and decisions: A review. Global Change Biology, 23(1): 28-41.

Scholes R J, 2016. Climate change and ecosystem services. Wiley Interdisciplinary Reviews: Climate Change, 7(4): 537-550.

Sonter L J, Johnson J A, Nicholson C C et al., 2017. Multi-site interactions: Understanding the offsite impacts of land use change on the use and supply of ecosystem services. Ecosystem Services, 23: 158-164.

Sturck J, Poortinga A, Verburg P H, 2014. Mapping ecosystem services: The supply and demand of flood regulation services in Europe. Ecological Indicators, 38: 198-211.

Sturck J, Schulp C J E, Verburg P H, 2015. Spatio-temporal dynamics of regulating ecosystem services in Europe: The role of past and future land use change. Applied Geography, 63: 121-135.

Sun F X, Lü Y H, Fu B J et al., 2016. Hydrological services by mountain ecosystems in Qilian Mountain of China: A review. Chinese Geographical Science, 26(2): 174-187.

Thom D, Rammer W, Seidl R, 2017. Disturbances catalyze the adaptation of forest ecosystems to changing climate conditions. Global Change Biology, 23(1): 269-282.

Trenberth K E, 2011. Changes in precipitation with climate change. Climate Research, 47(1/2): 123-138.

USDA, 1985. National Engineering Handbook, Section 4, Hydrology. United States Department of Agriculture, Soil and Conservation Service, Washington, DC.

Walz A, Braendle J M, Lang D J et al., 2014. Experience from downscaling IPCC-SRES scenarios to specific national-level focus scenarios for ecosystem service management. Technological Forecasting and Social Change, 86: 21-32.

Watson K B, Ricketts T, Galford G et al., 2016. Quantifying flood mitigation services: The economic value of Otter Creek wetlands and floodplains to Middlebury, VT. Ecological Economics, 130: 16-24.

Wang P T, Zhang L W, Li Y J et al., 2017. Spatio-temporal characteristics of the trade-off and synergy relationships among multiple ecosystem services in the upper reaches of Hanjiang River Basin. Acta Geographica Sinica, 72(11): 131-142. (in Chinese)

Yang C, Wang N, Wang S, 2017. A comparison of three predictor selection methods for statistical downscaling. International Journal of Climatology, 37(3): 1238-1249.

Yang L, Zhang L, Li Y et al., 2015. Water-related ecosystem services provided by urban green space: A case study in Yixing City (China). Landscape and Urban Planning, 136: 40-51.

Yuan Q, Wu S, Dai E et al., 2017. NPP vulnerability of the potential vegetation of China to climate change in the past and future. Journal of Geographical Sciences, 27(2): 131-142.

Zhang B, Li W H, Xie G D et al., 2010. Water conservation of forest ecosystem in Beijing and its value. Ecological Economics, 69(7): 1416-1426.

Zhang L, Lü Y, Fu B et al., 2017. Uncertainties of two methods in selecting priority areas for protecting soil conservation service at regional scale. Sustainability, 9(9): 1577.

Zuo D, Xu Z, Yao W et al., 2016. Assessing the effects of changes in land use and climate on runoff and sediment yields from a watershed in the Loess Plateau of China. Science of the Total Environment, 544: 238-250. 